\title{
Human Odorant Binding Protein 2a has Two Affinity States and is Capable of Binding Some Uremic Toxins
}

\author{
Whitson $\mathrm{KB}^{1^{*}}$ and Whitson $\mathrm{SR}^{2}$ \\ ${ }^{1}$ Department of Physics, Geology, and Astronomy, University of Tennessee, Chattanooga, TN, 37403, USA \\ ${ }^{2}$ Department of Chemistry, University of Tennessee, Chattanooga, TN, 37403, USA
}

"Corresponding author: Whitson KB, Department of Physics, Geology, and Astronomy, University of Tennessee, Chattanooga, TN, 37403, USA, Tel: 423-425-1766; Email: Kristin-Whitson@utc.edu

Received date: August 30, 2014; Accepted date: October 09, 2014; Published date: October 16, 2014

Copyright: ${ }^{\odot} 2014$ Whitson KB, et al. This is an open-access article distributed under the terms of the Creative Commons Attribution License, which permits unrestricted use, distribution, and reproduction in any medium, provided the original author and source are credited.

\begin{abstract}
Human odorant binding proteins (OBPs) are lipocalins proposed to function by carrying small molecules through aqueous environments to olfactory receptors. In contrast to previous reports, results from fluorescence assays herein show that ligands bind to OBP-2a with two affinities, one with a micromolar and one with a nanomolar equilibrium dissociation constant. Computational modeling of the protein reveals these states could be associated with two binding sites for hydrophobic and/or aromatic molecules, and is not dependent on functional groups such as aldehyde moieties. Small-molecule hydrophobic uremic toxins like p-cresol were found to effectively compete for binding to OBP-2a nearly as well as traditional odorants like vanillin. The results support a possible molecular mechanism for interference of uremic toxins that could result in the impaired olfactory sensitivity described for patients with advanced renal disease.
\end{abstract}

Keywords: Odorant binding protein; Lipocalin; Fluorescence; Uremic toxins; Olfaction

\section{Introduction}

Human olfaction is characterized by the ability to detect and discriminate between thousands of distinct odors; however, the specific molecular mechanism by which much of the pathway occurs is not elucidated. Sensory perception is triggered by binding of an odorant ligand to G-protein coupled olfactory receptors on the surface of neurons within the nasal epithelium. This interaction initiates a signal transduction cascade with well-known downstream molecular components that convert the chemical stimulus to an electrical signal and neuronal response interpreted by the brain [1]. Within the olfactory epithelium, olfactory receptor neurons are embedded in a dense layer of mucus that presents a barrier to vapors of volatile odorants in reaching the G-coupled receptor. Odorants are slowed in diffusion across the mucus and can become trapped or degraded if they interact with soluble macromolecules there [2]. Accordingly, a volatile odorant with low water solubility or relatively high hydrophobicity is expected to have a very low diffusion constant in the nasal mucosa.

Binding of ligand to receptor may occur directly or via ligand interaction with odorant binding proteins (OBPs), small non-specific carrier proteins localized in the nasal mucosa. These proteins perform an intermediate step of reversibly binding small hydrophobic odorants to shuttle them across the aqueous mucus layer to the olfactory epithelium and deliver them to the olfactory receptors [2]. Because the entropic cost of diffusion for hydrophobic odorants across aqueous mucus is presumably high, OBPs must act as a catalyst to provide a mechanism for shuttling of ligand to receptor. Ultimately, OBPs function by controlling the concentration of odorants available to embedded receptors in the olfactory epithelium. Direct interaction of OBPs with the G-coupled receptor has not been conclusively established; however, binding of OBPs to specific hydrophobic odorants has been shown $[3,4]$. In addition to their role in shuttling odorants across aqueous layers to receptors, OBPs may control odorant availability by post-signal transduction sequestration events to allow for desensitization of receptors.

OBPs have been identified in a variety of species [5]. In vertebrates, they are low molecular weight proteins $(\sim 20 \mathrm{kDa})$ that have previously been reported to bind odorants with dissociation constants in the micromolar range $[3,4]$. Vertebrate OBPs are structurally classified as lipocalins; members of this superfamily display low sequence identity but share a three-dimensional protein structure consisting of an eightstranded beta-barrel with a hydrophobic cavity flanked by a short alpha-helix at the C-terminus of the protein [6]. In humans, three putative odorant binding proteins have been identified [3]. The mRNA encoding these proteins is expressed in brain, heart, kidney, liver, genital, and lung tissues, in addition to expected localization in the oral sphere $[7,8]$. To date, only OBP-2a and tear lipocalin have been characterized on a protein level. OBP-2a is a soluble carrier protein that is $45 \%$ homologous to rat OBP-2, has been isolated from human nasal mucosa, and has been demonstrated to bind odorants of different chemical classes, the strongest affinity being demonstrated for long-chain aldehydes and fatty acids [3,4]. While it has been noted that the binding cavity in the beta-barrel structure is large enough to accommodate multiple ligands [9], only one affinity state has been reported for OBP-2a [3,4].

Human thresholds toward a particular odorant are typically normal distributions, with one extreme representing enhanced sensitivity, and the other, specific anosmia toward the odor. The range of anecdotal discrepancies in non-diseased humans could be a result of single nucleotide polymorphisms in G-coupled olfactory receptors [10]. Regulation of the perceived signal may also be achieved through spatial coding within the olfactory epithelium or olfactory bulb of the brain, or by the timing of action potentials $[11,12]$. Space coding and 
ligand-receptor combinatorial mechanisms, in particular, could be enhanced by the inclusion of OBPs into the presentation mechanism.

Anosmia has been investigated as a symptom of some human diseases including neurodegenerative disorders (Alzheimer's and Parkinson's disease [13]) and chronic conditions (Chronic Kidney Disease, CKD, and End-stage Renal Disease, ESRD) [14]. In neurodegenerative diseases, impaired cognitive function and defects appear to correlate with loss of olfaction and the ability to discriminate between odors. This pathology is likely related to changes in the olfactory bulb of the brain. In ESRD patients, it is unlikely that impaired olfaction is related to cognitive decline. Patients do not experience an increase in odor perception ability after undergoing haemodialysis, but fully regain olfactory function after renal transplantation. This finding has been suggested to indicate that reduced olfaction is due to buildup of uremic toxins not cleared by current dialysis techniques [14]. Most uremic toxins are small metabolic degradation products, many of which (e.g. phenols, indoles, pyrimidines, and nucleotide bases) have hydrophobic character. In addition to these degradation products, toxic accumulation of some low-molecular weight proteins occurs. Notably, retinol binding protein is one of these [15], a lipocalin closely structurally related to OBPs. We propose herein that in CKD and ESRD patients, hydrophobic uremic toxins may bind to OBPs and related lipocalins, thus saturating the olfactory signaling pathway at this level and inhibiting OBPs from participating in normal transport and delivery functions for other odorants to olfactory receptors.

\section{Materials and Methods}

\section{Materials}

Unless otherwise noted below, all reagents used in protein production and fluorescence studies were purchased from SigmaAldrich or Fischer Scientific, and were certified ACS reagent grade or better.

\section{Protein expression and purification}

The cDNA clone for human OBP-2a was purchased from OriGene (Rockville, MD) and subcloned into the pSV278 bacterial expression vector (kindly provided by Vanderbilt University). Use of this expression vector allows for creation of recombinant OBP-2a that contains N-terminal maltose-binding protein (MBP) and His6 tags used to facilitate protein purification. Sequencing of the construct (Vanderbilt University core facility) verified accuracy of the expression plasmid.

The vector was transfected into BL21 or pLys-S strains of Escherichia coli and grown in $\mathrm{LB}$ rich broth media at $37^{\circ} \mathrm{C}$. All cultures were supplemented with $50 \mu \mathrm{g} / \mathrm{mL}$ kanamycin for selection of stably transfected bacteria. Cultures reaching an optical density at 600 $\mathrm{nm}$ of about 1.0 were cooled on ice water, then induced with $1.0 \mathrm{mM}$ isopropyl- $\beta$-D-thiogalactopyranoside and grown for 5 hours at $25^{\circ} \mathrm{C}$. Cells were collected by centrifugation and lysed by sonication for 2-3 minutes in 20 second bursts. Bacterial cell lysates with fusion protein were applied to an amylose resin column (which binds MBP), washed with $20 \mathrm{mM}$ tris-hydroxymethyl-aminomethane (Tris- $\mathrm{HCl}$ ), $200 \mathrm{mM}$ $\mathrm{NaCl}$, and $1 \mathrm{mM}$ EDTA column buffer, and eluted in column buffer supplemented with $10 \mathrm{mM}$ maltose. Protein that was eluted from the amylose resin was further purified by incubation with Ni-NTA resin (which binds the fusion protein His6-tag sequences) in the presence of
$20 \mathrm{mM}$ imidizole for one hour followed by a wash with $50 \mathrm{mM}$ imidizole to remove non-specific interactions. Elution from the $\mathrm{Ni}$ NTA affinity column was done with column buffer containing 250 $\mathrm{mM}$ imidizole. Subsequent sodium dodecyl sulfate-polyacrylamide gel electrophoresis and Western blotting using an anti-His6 tag as the primary antibody revealed relatively pure samples (>90\%) of the MBPOBP-2a fusion protein. To account for non-specific binding interactions in fluorescence binding studies that resulted only from interaction with the MBP portion of the fusion protein, we expressed the $\mathrm{N}$-terminal region of the fusion protein (containing the MBP and His6 tag sequences but excluding the OBP-2a sequence) from the pSV278 vector and purified the MBP protein using an identical protocol to that described above.

\section{Fluorescence binding experiments}

Binding assays to measure dissociation constants were performed similarly to those by Tcatchoff et al. [4]. For saturation binding experiments, a $2 \mu \mathrm{M}$ solution of MBP-OBP-2a fusion protein was made in $50 \mathrm{mM}$ Tris buffer, $\mathrm{pH}$ 7.4. N-phenyl-1-naphthylamine (NPN) was successively titrated into the protein solution and binding was allowed to reach equilibrium by incubation for 5 minutes at room temperature before data acquisition. Less than $5 \%$ of the total volume of analyzed sample was from the NPN stock (made in methanol) at any concentration. Fluorescence excitation and emission scans were acquired with a Cary Eclipse fluorescence spectrometer for NPN in the presence or absence of MBP-OBP-2a and for the protein alone in order to identify wavelengths for excitation and emission of the fluorophore that reflected changes upon interaction with protein. Consistent with the previous findings of Tcatchoff et al. [4], maximum changes in the fluorescence spectra resulting from binding occurred when exciting the NPN fluorophore at $337 \mathrm{~nm}$ and monitoring its emission at $410 \mathrm{~nm}$. Identical experiments using the MBP fusion protein were performed side-by-side with the MBP-OBP-2a experiments in order to determine non-specific binding interactions occurring as a result of the fusion sequence used. Backgroundsubtracted data were analyzed with SigmaPlot 12 (Systat Software) using one-site saturation binding model of the form: $y=\left(\operatorname{ymax}^{*} \mathrm{x}\right) /(\mathrm{x}$ $+\mathrm{KD})$, or the equivalent two-site saturation binding model, where KD is the equilibrium dissociation constant for NPN binding to OBP-2a, fluorescence is plotted on the abscissa, and the concentration of NPN is the ordinate.

For competition binding experiments, NPN was allowed to equilibrate with $2 \mu \mathrm{M}$ protein (either MBP-OBP-2a or the MBP control). Competition of octanal and vanillin was performed against either 20 or $65 \mu \mathrm{M} \mathrm{NPN}$; competition of all other ligands was performed against $20 \mu \mathrm{M}$ NPN. In the experiments, increasing concentrations of competitor ligands were successively titrated into the sample; the volume of sample increased by less than $5 \%$ throughout the experiment. At each concentration, a measurement of the fluorescence emission of NPN was taken. Data were plotted as fluorescence intensity vs. concentration of competitor, and fit to oneor two-site competition binding models to determine the IC50, from which the Ki was calculated. The following equations and models were used: $y=y m i n+[(y m a x-y m i n) /(1+10 \log (x)-\log ($ IC50) $)]$ (where ymax is the total fluorescence and ymin is the fluorescence due to nonspecific binding $)$, and $\mathrm{Ki}=\mathrm{IC} 50 /[1+([\mathrm{NPN}] / \mathrm{KD}$ of NPN $)]$. 


\section{Computational modeling}

Molecular models of the tertiary structure of wild-type OBP-2a were made with the Swiss-model server [16] by alignment of the sequence with the solved structures of human tear lipocalin (PDB ID: 1XKI and 3EYC) [9,17]. Ligands were built and docked into the structures using Scigress Explorer (Fujitsu), where the active site was defined as all residues whose side-chains were interior to the betabarrel. Energies of binding were calculated with Scigress and force fields analyzed with Swiss-PDB viewer.

\section{Results and Discussion}

\section{Protein production}

The protein construct employed in binding studies contained a sequence identical to the wild-type sequence of human OBP-2a. In contrast, previous reports were based on a construct of OBP-2a containing Cys114 to Ser and Thr163 to Ala (numbered according to the full-length sequence) that was made to prevent heterogeneity of the product due to post-translational alkylation or O-linked glycosylation when expressed in Pichia pastoris [4]. By further analysis of lysine mutants, Tcatchoff et al. [4] reported that Lys127 was the major determinant of binding to aldehyde moieties. Notably, models of the three-dimensional structure of OBP-2a (herein and in other studies $[3,4,18])$ show that Cys114 is proximal to residues within the binding pocket for aldehyde groups ( $\sim 6.6 \AA$ from the Cys sulfhydryl group to the side-chain amino group of Lys 127 that reportedly forms a Schiff base in binding interactions with aldehyde odorants [18]). The protein construct used in studies herein was derived from a protein expression method developed for bacterial cells that allows for purification from cell lysates by a chimeric fusion with maltosebinding protein (MBP) and a His6 tag sequence. We also generated a $\mathrm{MBP}$ fusion sequence protein that lacked the OBP-2a sequence for use as a control in fluorescence experiments reported below.

\section{Fluorescence binding studies}

The study of binding interactions between OBP-2a and ligands described herein uses fluorescence spectroscopy as the primary tool. Previous reports showed N-phenyl-1-napththylamine (NPN) binds to OBP-2a and can be used to assess ligand affinity by competition binding experiments [4]. NPN is a fluorescent dye whose quantum yield dramatically increases in hydrophobic environments; thus, it fluoresces when bound to the hydrophobic cavity in OBP-2a, but emission intensity is minimal when NPN is free in aqueous solution. As shown in Figure 1, when NPN was incubated with the MBPOBP-2a protein construct, an excitation and emission peak appeared in the spectra with maxima around $340 \mathrm{~nm}$ and $415 \mathrm{~nm}$, respectively, consistent with binding of the fluorophore to a hydrophobic pocket in the protein.

Saturation binding experiments for NPN were performed to show consistency of the protein construct used in our studies with those previously reported [4]. In these experiments, fluorescence emission intensities were collected for the chimeric MBP-OBP-2a protein with successive titrations of NPN into the sample over a concentration range of $5 \mathrm{nM}$ to $40 \mu \mathrm{M}$. Analogous experiments were performed for NPN with the MBP fusion sequence to account for effects of nonspecific interactions with that portion of the chimeric protein. The background subtracted data (Figure 2) were fit to saturation binding models to determine an equilibrium dissociation constant $\left(\mathrm{K}_{\mathrm{D}}\right)$ for the interaction between NPN and OBP-2a.
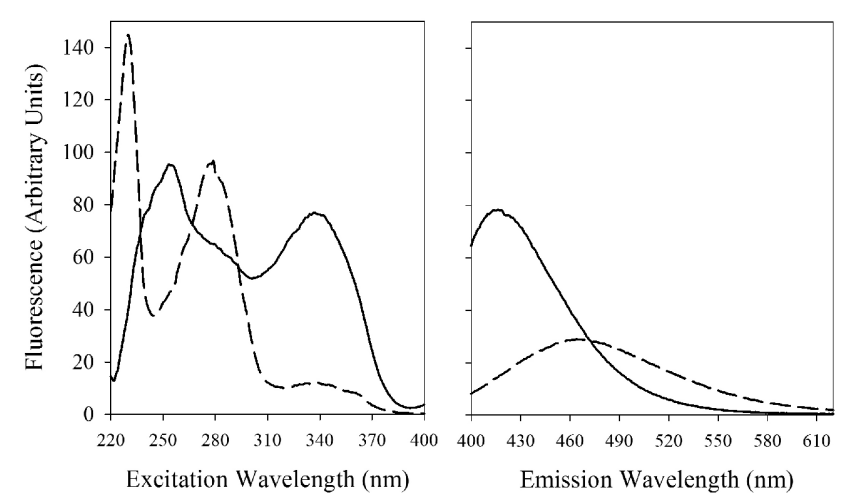

Figure 1: Fluorescence emission and excitation scans for NPN in the presence and absence of OBP-2a. Excitation spectra for $4 \mu \mathrm{M}$ NPN in the presence of $2 \mu \mathrm{M}$ MBP-OBP-2a (solid line) or $2 \mu \mathrm{M}$ MBP portion of the protein construct (dashed line) were collected. For excitation scans, emission was monitored at $410 \mathrm{~nm}$. For emission scans, the sample was excited with $337 \mathrm{~nm}$ light. Notably, upon binding to a hydrophobic cavity in MBP-OBP-2a, a dramatic blue-shift in the peak emission wavelength and an increase in fluorescence intensity occur.

The fluorescence intensity curve over the full range of tested NPN concentrations revealed the presence of two plateaus; thus, data were fit to both a one-site and two-site saturation models to distinguish between possible different modes of binding. For a one-site fit, the analysis yielded a $\mathrm{KD}$ of $14 \pm 2 \mu \mathrm{M}(\mathrm{P}<0.0001, \mathrm{R} 2=0.9900)$. A two-site saturation binding model for the full data set did not provide a statistically-significant fit for interaction of NPN with a second, loweraffinity OBP-2a binding site due to low data density in the concentration range that would define this site. It is possible that the presence of a lower-affinity binding site reflects binding of the probe to small hydrophobic areas on the surface of OBP-2a at these higher concentrations. NPN/OBP-2a equilibrium binding studies in previous reports [3,4] did not describe data generated using NPN concentrations greater than $30 \mu \mathrm{M}$; however, they did show by mutational analysis that NPN served as a probe for the interior hydrophobic binding cavity in OBP-2a. Therefore, collected data for MBP-OBP-2a and the MBP portion of the fusion construct were reanalyzed to include only the concentrations of NPN that described binding affinities or interactions with the interior hydrophobic cavity that were previously reported (less than $30 \mu \mathrm{M}$ ). The new analysis was again only statistically significant for the one-site fit (Figure 2), yielding a $\mathrm{K}_{\mathrm{D}}$ of $7.9 \pm 0.8 \mu \mathrm{M}(\mathrm{P}<0.0001, \mathrm{R} 2=0.9985)$. Comparison of this dissociation constant to the previously-reported single-site fit $\mathrm{K}_{\mathrm{D}}$ of $3 \pm 2 \mu \mathrm{M}$ [4] shows good agreement and indicates that the structural integrity and folding of the OBP-2a portion of the protein construct was not compromised by inclusion of the fusion sequences. The slightly higher affinity reported by Tcatchoff et al. might result from the presence of a C114S mutation on the interior of the hydrophobic binding pocket proximal to the reported determinant residue for binding. The results of our experiments could suggest that this additional mutation played a role in increasing the affinity of the protein for the specific ligand NPN. 


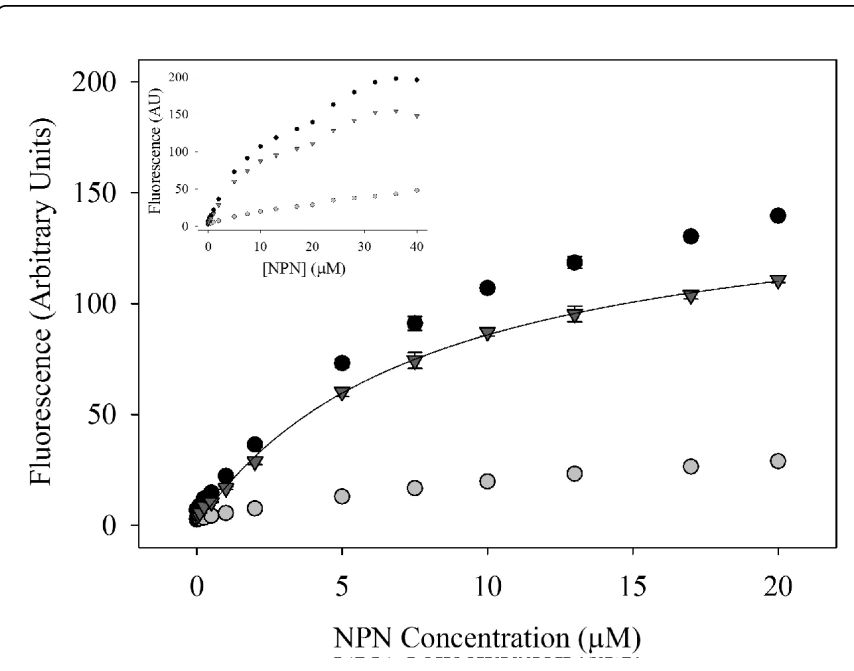

Figure 2: Saturation binding experiment for NPN with OBP-2a. Successive amounts of NPN were titrated into protein samples in parallel experiments. Data for the control MBP portion of the protein construct (gray circles) was subtracted from data for the MBP-OBP-2a fusion protein (black circles) to yield the specific binding of NPN to OBP-2a (gray triangles). Data from the full range ( $5 \mathrm{nM}$ to $40 \mu \mathrm{M} \mathrm{NPN}$ ) is shown in the graph inset; data that was fit with saturation binding models to recover equilibrium binding constants ( $5 \mathrm{nM}$ to $20 \mu \mathrm{M} \mathrm{NPN}$ ) is shown in the full display. Error bars represent the standard deviation of three independent trials, and the fit shown is for a one-site model (black line).

Competition binding assays were used to measure the ability of particular odorants to displace NPN from OBP-2a in order to infer the affinity for various odorants for the NPN binding site. Since previous reports indicated OBP-2a was specific for aldehydes with a hydrophobic character, we performed studies with octanal (an eightcarbon aldehyde) and vanillin (a phenolic aldehyde). In these experiments, a saturating dose of NPN for the hydrophobic binding cavity $(20 \mu \mathrm{M})$ was allowed to equilibrate with protein, and the fluorescence emission of NPN was monitored as increasing concentrations of competitor ligands were successively titrated into the sample. Background fluorescence readings from the MBP fusion portion of the protein were subtracted from those of the MBP-OBP-2a samples, and data were fit to one- and two-site competition binding models to recover binding parameters. The model assumes NPN and the competitor odorant both bind reversibly to the same binding site; recovered parameters include the $\mathrm{IC}_{50}$ and the $\mathrm{K}_{\mathrm{i}}$.

As shown in Figure 3, a decrease in fluorescence emission intensity of NPN occurred when octanal was added to the sample, indicating the odorant outcompeted NPN for binding. Fits to the data with a two-site model were statistically better than with a one-site model (yielding non-overlapping ranges for $\mathrm{K}_{\mathrm{i}}$ values and $\mathrm{P}<0.0001$ in all cases) for all odorants with an aromatic or hydrophobic nature tested (Table 1). This result suggests that OBP-2a has at least two binding sites for competing odorants against NPN, where competitors have either high- or low-affinity interactions with the protein. The binding analysis for NPN only yielded one affinity state; however, NPN (with a three-ringed aromatic structure) may occupy more volume in the hydrophobic binding pocket of OBP-2a, thus being stericly hindered from having more than one mode of interaction with the protein, in contrast to the two modes of binding seen for smaller ligands.

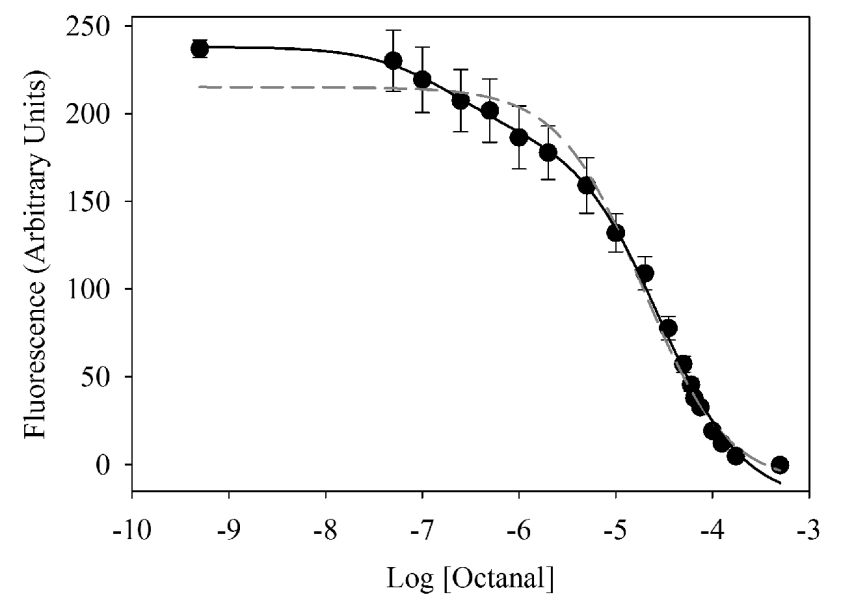

Figure 3: Competition binding of octanal with NPN to OBP-2a. Successive amounts of octanal were added to protein samples of MBP-OBP-2a or the control MBP that was previously incubated with saturating concentrations of NPN. Background-subtracted data is shown with error bars representing one standard deviation of three independent experiments. Data were fit by a one-site (gray dash) or two-site (black line) model to recover binding parameters. The one-site competition model yielded an IC50 of $30 \mu \mathrm{M}(\mathrm{R} 2=$ 0.9552 , and $\mathrm{P}<0.0001)$; the two site model yielded an IC50 for site 1 of $69 \pm 6 \mu \mathrm{M}$ and for site 2 of $0.38 \pm 0.08 \mu \mathrm{M}$, with $70 \%$ of ligands filling site 1 . The two sites defined by the data are statistically significant $(\mathrm{P}<0.0001)$, and the $\mathrm{R} 2$ value is expectedly better (0.9979) than for the one-site fit.

Alternatively, the data could suggest that NPN has equivalent equilibrium interactions with two distinct binding sites in the core of the protein, thus only one affinity state is observed. The presence of a high-affinity binding site for competitor ligands was not previously noted for OBP-2a; however, data density shown in former publications $[3,4]$ was low around the high-affinity site found in present studies, thus this site may have gone undetected. For competitor ligands, the low-affinity site is of micromolar affinity, is bound by one-third or more of the ligand population in solution, and may be more solventaccessible. The nanomolar affinity site for competitors may be buried deeper in the core of the protein. The solvent accessibility is suggested by the competition binding data for isoamyl acetate (a small hydrophilic ester) that displays a single micromolar binding affinity, but is only able to displace $30 \%$ of the total bound NPN. Repetition of the competition binding experiment for vanillin and octanal at a supersaturating dose of NPN $(65 \mu \mathrm{M})$ did not give significantly different values for the binding constants of vanillin competing against $20 \mu \mathrm{M}$ NPN shown in Table 1 . 


\begin{tabular}{|l|l|l|l|l|}
\hline Molecule & $\mathrm{K}_{\mathrm{i} 1}(\mu \mathrm{M})$ & $\mathrm{K}_{\mathrm{i} 2}(\mu \mathrm{M})$ & $\%$ of ligands occupying site 1 & Minimum Fluorescence $(\%$ of max $)$ \\
\hline Octanal & $7 \pm 1^{\mathrm{b}}$ & $0.041 \pm 0.008$ & $70 \pm 2$ & $8 \pm 1$ \\
\hline Vanillin & $3.4 \pm 0.4$ & $0.022 \pm 0.008$ & $80 \pm 2$ & $0 \pm 1$ \\
\hline Isoamyl acetate ${ }^{\mathrm{a}}$ & $8 \pm 1$ & --- & 100 & $70 \pm 3$ \\
\hline$p$-cresol & $8 \pm 2$ & $0.034 \pm 0.006$ & $40 \pm 2$ & $31 \pm 1$ \\
\hline 2-amino-p-cresol & $14 \pm 3$ & $0.03 \pm 0.01$ & $49 \pm 3$ & $28 \pm 2$ \\
\hline Cytosine & $11 \pm 3$ & $0.042 \pm 0.009$ & $37 \pm 2$ & $34 \pm 1$ \\
\hline Thymine & $9 \pm 3$ & $0.03 \pm 0.01$ & $42 \pm 4$ & $37 \pm 2$ \\
\hline Uracil & $14 \pm 6$ & $0.042 \pm 0.01$ & $30 \pm 4$ & $33 \pm 2$ \\
\hline $\begin{array}{l}\text { aData for isoamyl acetate were best fit by a one-site model. } \\
\text { bErrors represent the standard error in the competition binding model fits. }\end{array}$ & \\
\hline
\end{tabular}

Table 1: Constants recovered from the two-site competition binding model for interactions of selected small molecules competing with $20 \mu \mathrm{M}$ NPN for binding to OBP-2a.

Because anosmia has been reported as a symptom of the uremic condition and OBP-2a is observed to bind small hydrophobic molecules with an aromatic nature, we performed competition binding assays to investigate possible interactions of known aromatic uremic toxins (such as $p$-cresol) and other products of protein and nucleic acid degradation (cytosine, thymine, and uracil) with OBP-2a. Uremic toxins are defined as abundant small molecular-weight species that accumulate and remain in patients with renal disease even after that patient has undergone dialysis [15]. The data (Figure 4) show that these hydrophobic uremic toxins can compete with NPN, and possibly other odorants, for binding to OBP-2a. For instance, recovered Ki values indicate that a larger fraction of $p$-cresol interacts with the highaffinity binding site to OBP-2a than does vanillin, an odorant with a very similar chemical structure. As shown in Table 1, all uremic toxins tested bound with the same affinity classes (micro and nano-molar sites) as the aldehydes tested, and in general, more are distributed in the high-affinity binding site $(\sim 60 \%)$ than the low-affinity site. The relatively high concentration of toxins in the bloodstream and kidneys in the disease state (e.g., $186 \mu \mathrm{M}$ for $p$-cresol [15]) also suggests the possibility that they could out-compete other odorants for binding sites on OBPs. In this case, if a toxin associates with an OBP, the OBP may be inhibited from performing its normal function in capturing odorants (like vanillin) to transport to olfactory receptors.

We did not observe specific functional groups for $p$-cresol (e.g., the amine group of 2 -amino-p-cresol) to affect competitor binding specificity to the protein, possibly indicating that ligand size or shape plays a larger role in binding affinity for small toxins to OBPs than particular constituent functional groups. Conversely, the uremic toxins tested do not displace as large a fraction of the total bound NPN from OBP-2a as do the aldehyde odorants tested ( $70 \%$ vs. $>90 \%)$, possibly correlating with the energetic balance between formation of Schiff base interactions between Lys127 at the bottom of the betabarrel structure of OBP-2a with aldehydes [18], the absence of the aldehyde moiety on tested uremic toxins, and the increased size of the NPN molecule (three phenyl rings) that buries a larger surface area in the hydrophobic cavity of OBP-2a.

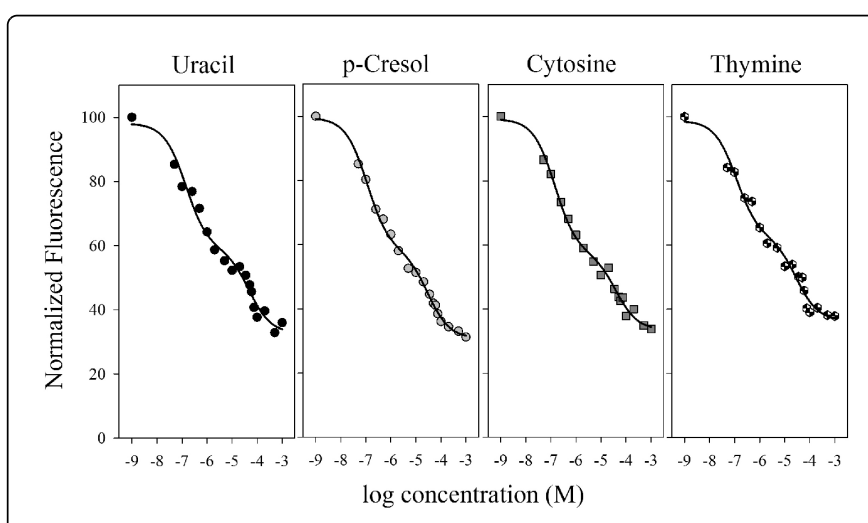

Figure 4: Competition binding of selected uremic toxins against NPN for OBP-2a. Successive amounts of uremic toxin were titrated into protein samples of MBP-OBP-2a or the control MBP that was previously incubated with $20 \mu \mathrm{M}$ NPN. The average of three trials of the background-subtracted data is shown for each competitor with the two-site competition binding fit (black line). Recovered $\mathrm{K}_{\mathrm{i}}$ values and other fitting parameters are given in Table 1.

Consistent with the results of isoamyl acetate studies, if binding interactions with OBP-2a are made in a more solvent accessible way (nearer the outside of the beta-barrel), binding affinities of toxins and NPN would be independent, but the displacement would be less complete. Such would be the case for a less restrictive carrier protein than has previously been suggested [4], where binding sites for different molecules overlap somewhat but are slightly different. This hypothesis is also consistent with data in previous reports showing a greater fraction of fluorescent probe is displaced by some odorants when a fluorophore with only two aromatic rings is used (e.g., $\alpha$ pinene displaced $25 \%$ of bound NPN, but $40 \%$ of bound $11-((5-$ (dimethylaminonaphthalenyl-1-sulfonyl)amino)undecanoic acid) $[3,4]$. 


\section{Computational modeling}

To further investigate possible binding sites and modes of interaction, we modeled the molecular structure of hOBP-2a based on alignment to the solved structure of human tear lipocalin, which is $65 \%$ similar in sequence. The overall protein architecture consists of an eight-stranded antiparallel beta-barrel with an attached alpha-helix typical of the lipocalin structural superfamily. The model showed a wide binding cavity within the beta-barrel, the entrance to which is formed by disordered loops connecting neighboring beta strands; these disordered loop regions are least conserved in the primary sequence between OBP-2a and tear lipocalin. Overall, the structural features of the protein are suggestive of promiscuous binding behavior between human OBP-2a and hydrophobic odorant molecules, particularly due to the wide binding pocket, as noted for structures of other proteins in this family [9].

Ligands used in molecular docking studies included all those with experimental data shown in Table 1 in addition to the uremic toxin indoxyl sulfate and the amino acids tryptophan and tyrosine (precursors to indoxyl sulfate and $p$-cresol in protein metabolism, respectively). We defined the active site for ligand binding as all residues with side chains interior to any portion of the beta-barrel in order to provide the widest variety of energy sampling conformations to docking ligands and eliminate forced effects of presumed specific interactions (for example with Lys127, the binding determinant previously reported to direct specificity for aldehydes [4]). The results show that all hydrophobic ligands found energy minima within the cavity and associated energies of the ligand/protein complex were negative (between -150 and $-250 \mathrm{kcal} / \mathrm{mol}$ in vacuo), indicating stable interactions within the binding pocket. Further analysis of the ligandbound structures and a more detailed force field analysis suggest that binding of the ligands studied occurs primarily through van der Waals interactions to minimize entropic costs of solvation by water, consistent with their hydrophobic nature.

Additional docking computations were performed where a second ligand was docked to protein already bound with ligand in the cavity. These experiments showed that the interior of the beta-barrel could accommodate two molecules of any ligand tested. The overall energy of the double-ligand-bound complex was less negative (by $\sim 100 \mathrm{kcal} /$ $\mathrm{mol}$ ), but still stable. As shown in Figure 5, the energy minimum for the first ligand bound was located nearer residues at the opening of the barrel (like Leu62), while the second ligand found an energy minimum deeper in the cavity, more proximal to Lys127, regardless of the ligand docked. Docking calculations using three ligands yielded positive energy values, suggesting binding of more than two small ligands is energetically unfavorable and unstable.

For the computational models where two ligands were bound, the interaction between the ligand bound nearest the opening of the barrel was more stable than the second energy minimum found. The existence of two binding sites observed in fluorescence binding studies does match with the observation of the hydrophobic cavity accommodating two distinct ligands. However, specific physical binding sites cannot be correlated to the experimentally-observed affinity states; only direct observation of the on and off rates for each affinity class would provide information to separate the inherent ratios of the equilibrium dissociation constant. Alternatively, it is possible that the two affinity states observed do not correspond to different physical binding locations within the binding pocket, but instead correspond to different modes of interaction with the same site on the protein, with either the protein or the ligand having two distinct conformations in the ensemble solution measured in fluorescence competition assays.

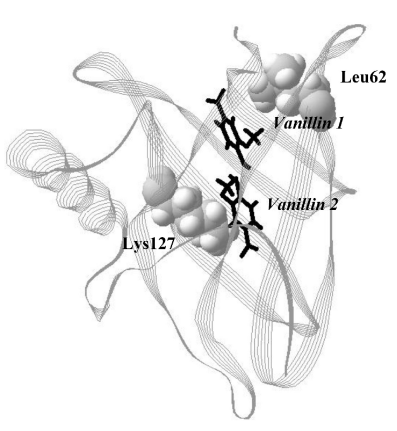

Figure 5: Homology model of OBP-2a with two ligands in energy minimized positions. Two vanillin molecules (black stick models) can be easily accommodated in the hydrophobic cavity of the betabarrel. Selected amino acids in close proximity to the docked ligands are in space-filling representation.

The existence of two distinct binding sites that could be simultaneously filled would suggest a different stoichiometry than has been previously reported for ligand-OBP-2a interactions. Isothermal titration calorimetry studies could verify the stoichiometry suggested. Lastly, there is a possibility that the linkage between the MBP fusion portion of the protein and the OBP-2a domain created an additional binding site that is observed via fluorescence studies and cannot be directly background-subtracted; further studies using the OBP-2a domain only would elucidate this possibility. On the other hand, because the high-affinity site for competition against NPN has thus far only been reported for OBP-2a using the MBP-OBP-2a construct herein, and because NPN showed only non-specific binding interactions to the MBP portion of the fusion construct (shown by a classic linear trend for non-specific binding in the law of mass action, as seen in Figure 2), this alternative is less likely.

An understanding of odorant recognition and molecular interactions of OBPs is not only important for elucidation of human olfactory signaling pathways, but also for design in bioengineering applications where sensors detect and bind specific hydrophobic molecules, and for chemical synthesis of odor molecules intended to elicit a certain response or human perception. Experimental and computational data herein suggest that there may be two binding sites, and mutation of residues other than lysine in the hydrophobic cavity (e.g. Cys114) also affects the molecular recognition surface for small molecules. In addition, the results show that OBPs are capable of binding hydrophobic uremic toxins. While OBP-2a has not been specifically reported to be present in the uremic milieu, related lipocalins such as retinol binding protein have been, and OBPs are not localized only to the oral sphere. Nonetheless, the data support a mechanism by which uremic toxins could saturate available OBPs, thereby preventing their participation in the transportation of other odorants, and resulting in the anosmia reported for patients with renal disease.

\section{Acknowledgement}

The authors thank Mrinalini Dixit and Charles Cobb (Vanderbilt University Medical Center) for technical assistance in protein 
Citation: Whitson KB, Whitson SR (2014) Human Odorant Binding Protein 2a has Two Affinity States and is Capable of Binding Some Uremic Toxins. Biochem Anal Biochem 3: 156. doi:10.4172/2161-1009.1000156

Page 7 of 7

production, recognize the contributions of Amber Lane, Bomi Kim, and Joseph Kennedy in data collection, and appreciate James Pino's work in providing experimental support and helpful discussion. We are grateful for funding from the Wheeler Odor Research Center (K.B.W.) and the UTC Grote Chemistry funds (S.R.W.).

\section{References}

1. 1. Schild D, Restrepo D (1998) Transduction mechanisms in vertebrate olfactory receptor cells. Physiol Rev 78: 429-466.

2. 2. Pelosi P (2001) The role of perireceptor events in vertebrate olfaction. Cell Mol. Life Sci. 58: 503-509.

3. 3. Briand L, Eloit C, Nespoulous C, Bezirard V, Huet J, et al. (2002) Evidence of an odorant-binding protein in the human olfactory mucus location, structural characterization, and odorant-binding properties. Biochemistry, 41: 7241-7252.

4. 4. Tcatchoff L, Nespoulous C, Pernollet J, Briand L (2006) A single lysyl residue defines the binding specificity of a human odorant-binding protein for aldehydes. FEBS Lett. 580: 2102-2108.

5. 5. Tegoni M, Pelosi P, Vincent F, Spinelli S, Campanacci V, et al. (2000) Mammalian odorant binding proteins. Biochim. Biophys. Acta. 1482: 229-240.

6. 6. Flower D, North A, Sansom, C (2000) The lipocalin protein family: structural and sequence overview. Biochim. Biophys. Acta 1482: 9-24.

7. 7. Yanai I, Benjamin H, Shmoish M, Chalifa-Caspi V, Shklar M, et al (2005) Genome-wide midrange transcription profiles reveal expression level relationships in human tissue specification. Bioinformatics 21: 650-659.

8. 8. Lacazette E, Gachon A, Pitiot G (2000) A novel human odorantbinding protein gene family resulting from genomic duplicons at $9 \mathrm{q} 34$ differential expression in the oral and genital spheres. Hum. Mol. Genet. 9: 289-301.

9. 9. Breustedt D, Korndorfer I, Redl B, Skerra A (2005) The 1.8- $\AA$ crystal structure of human tear lipocalin reveals an extended branched cavity with capacity for multiple ligands. J. Biol. Chem. 280: 484-493.

10. 10. Keydar I, Ben-Asher E, Feldmesser E, Nativ N, Oshimoto A, et al. (2013) General olfactory sensitivity database (GOSdb): candidate genes and their genomic variations. Hum. Mutat. 34: 32-41.

11. 11. Laurent, G (2002) Olfactory network dynamics and the coding of multidimensional signals. Nature Rev. Neurosci. 3: 884-895.

12. 12. Johnson B, Leon $M$ (2007) Chemotopic odorant coding in a mammalian olfactory system. J. Comp. Neurol. 503: 1-34.

13. 13. Hawkes C. (2006) Olfaction in neurodegenerative disorder. Adv. Otorhinolaryngol. 63: 133-151.

14. 14. Raff A, Lieu S, Melamed M, Quan Z, Ponda M, et al. (2008) Relationship of impaired olfactory function in ESRD to malnutrition and retained uremic molecules. Am. J. Kidney Dis. 52: 102-110.

15. 15. Vanholder R, DeSmet R, Glorieux G, Argiles A, Baurmeister U, et al. (2003) Review on uremic toxins: classification, concentration, and interindividual variability. Kidney Int. 63: 1934-1943.

16. 16. Guex N, Peitsch M, Schwede T (2009) Automated comparative protein structure modeling with SWISS-MODEL and Swiss-PdbViewer: A historical perspective. Electrophoresis 30(S1): S162-173.

17. 17. Breustedt D, Chatwell L, Skerra A (2009) A new crystal form of human tear lipocalin reveals high flexibility in the loop region and induced fit in the ligand cavity. Acta Crystallogr. D. Biol Crystallogr. 65: 1118-1125.

18. 18. Charlier L, Cabrol-Bass D, Golebiowski J (2009) How does human odorant binding protein bind odorants? The case of aldehydes studied by molecular dynamics. C. R. Chimie 12: 905-910. 